
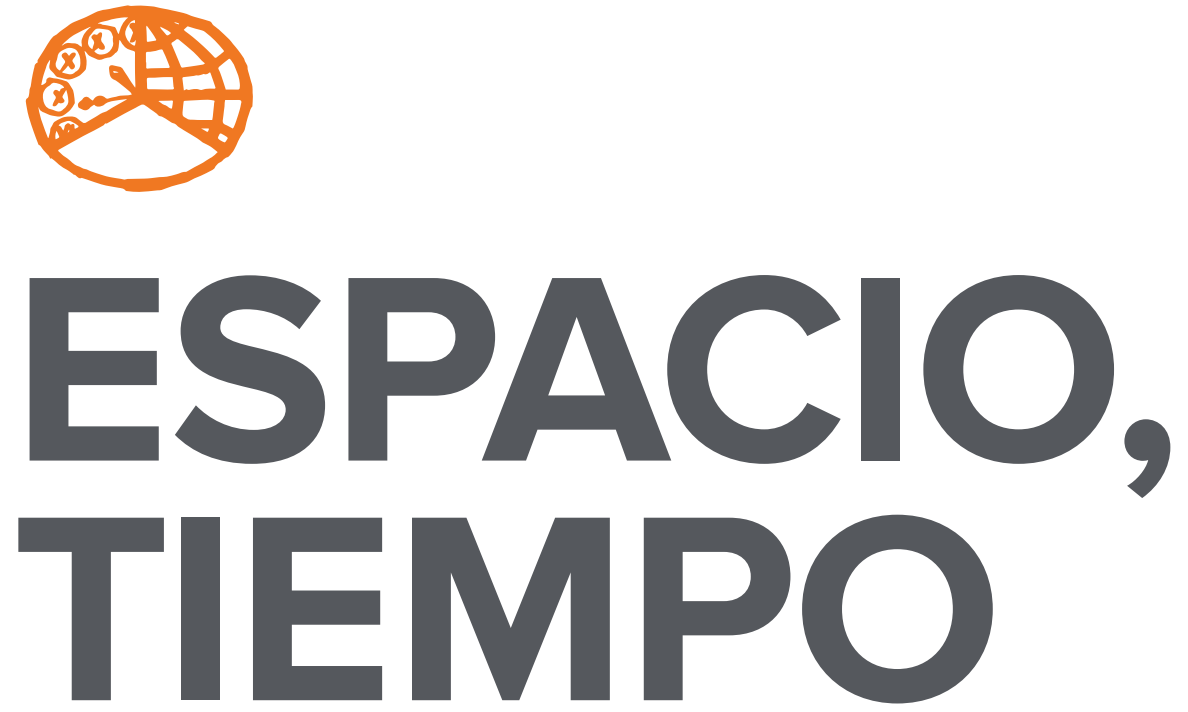

AÑO 2015

ISSN 1130-1082

E-ISSN 2340-1370
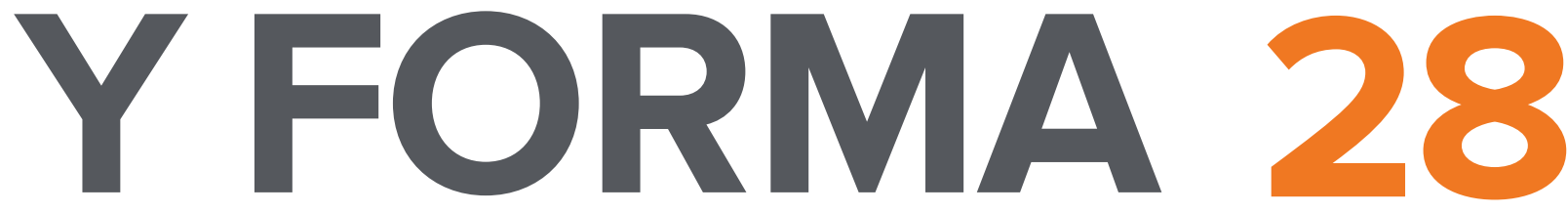

SERIE II HISTORIA ANTIGUA

REVISTA DE LA FACULTAD DE GEOGRAFÍA E HISTORIA 


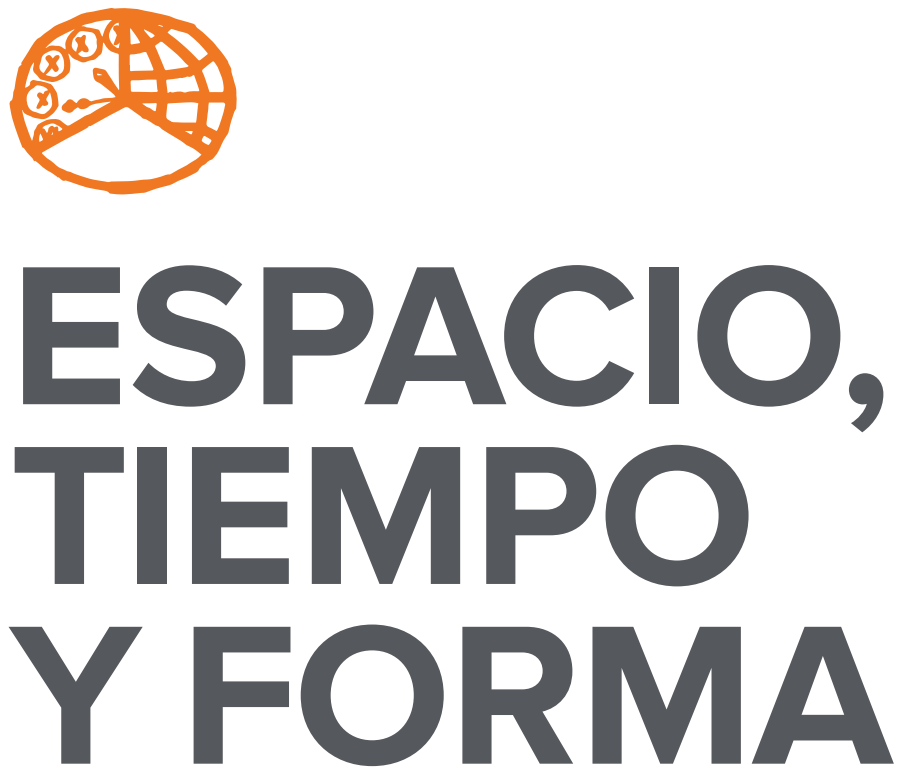

AÑO 2015

ISSN 1130-1082

E-ISSN 2340-1370

SERIE II HISTORIA ANTIGUA

REVISTA DE LA FACULTAD DE GEOGRAFİA E HISTORIA

http://dx.doi.org/10.5944/etfii.28.2015

\section{UกED}

UNIVERSIDAD NACIONAL DE EDUCACIÓN A DISTANCIA 
La revista Espacio, Tiempo y Forma (siglas recomendadas: ETF), de la Facultad de Geografía e Historia de la UNED, que inició su publicación el año 1988, está organizada de la siguiente forma:

$$
\begin{aligned}
& \text { SERIE I - Prehistoria y Arqueología } \\
& \text { SERIE II - Historia Antigua } \\
& \text { SERIE III - Historia Medieval } \\
& \text { SERIE IV - Historia Moderna } \\
& \text { SERIE V - Historia Contemporánea } \\
& \text { SERIE VI - Geografía } \\
& \text { SERIE VII - Historia del Arte }
\end{aligned}
$$

Excepcionalmente, algunos volúmenes del año 1988 atienden a la siguiente numeración:

$$
\begin{aligned}
& \mathrm{N} .^{\circ} 1 \text { - Historia Contemporánea } \\
& \mathrm{N}^{\circ} 2 \text { - Historia del Arte } \\
& \text { N. }{ }^{\circ} 3 \text { - Geografía } \\
& \mathrm{N} .^{\circ} 4 \text { - Historia Moderna }
\end{aligned}
$$

ETF no se solidariza necesariamente con las opiniones expresadas por los autores.

Espacio, Tiempo y Forma, Serie II está registrada e indexada, entre otros, por los siguientes Repertorios Bibliográficos y Bases de Datos: DICE, ISOC (CINDOC), RESH, IN-RECH, Dialnet, e-sPacio, UNED, CIRC, MIAR, FRANCIS, PIO, ULRICH'S, SUDOC, 2DB, ERIH (ESF).

\author{
UNIVERSIDAD NACIONAL DE EDUCACIÓN A DISTANCIA \\ Madrid, 2015 \\ SERIE II $\cdot$ HISTORIA ANTIGUA N. ${ }^{\circ} 28,2015$ \\ ISSN 1130-1082 - E-ISSN 2340-1370 \\ DEPÓSITO LEGAL \\ M-21.037-1988 \\ URL \\ ETF II · HISTORIA ANTIGUA $\cdot$ http://revistas.uned.es/index.php/ETFII \\ COMPOSICIÓN \\ Carmen Chincoa $\cdot$ http://www.laurisilva.net/cch \\ Impreso en España · Printed in Spain
}

(c) (7) (8) Esta obra está bajo una licencia Creative Commons

Reconocimiento-NoComercial 4.0 Internacional. 


\title{
MINIMA EPIGRAPHICA CLUNIENSIA
}

\section{MINIMA EPIGRAPHICA CLUNIENSIA}

\author{
Joaquín L. Gómez-Pantoja \& Mariano Rodríguez Ceballos \& Donato Fasolini \\ Recibido: 20/07/2015 - Aceptado: 10/09/2015 \\ DOI: http://dx.doi.org/10.5944/etfii.28.2015.15056
}

\section{Resumen}

Se describen a continuación siete inscripciones procedentes del Clunia Sulpicia (Coruña del Conde/Peñalba de Castro, Burgos); de ellas, cinco son inéditas y las otras dos se conocian pero sin edición completa. Cuatro de los letreros corresponden a grafitos sobre estuco, dos escritos en griego; ambas circuntancias son novedosas en este sitio arqueológico.

Palabras clave

Hispania Citerior; Clunia, epigrafía; graffiti; griego.

\begin{abstract}
This paper deals with some epigraphs found in Clunia Sulpicia (Coruña del Conde/ Peñalba de Castro, Burgos province, Spain). Five of them are new findings and the other two lacked a complete edition. It's worth noticing that four texts are graffiti, two written in Greek script.
\end{abstract}

\section{Keywords}

Roman Spain; Clunia; Epigraphy; Graffiti; Greek.

1. Universidad de Alcalá. Correos electrónicos: gomez.pantoja@uah.es; mrodriguezceballos@gmail.com; donato.fasolini@gmail.com. Grupo de investigación Oligarquías romanas de Occidente (ORDOAlcalá), Universidad de Alcalá, financiado con fondos públicos del Plan Nacional de Investigación Científica (HAR2011-29108-C04-02). Nuestro agradecimiento a Alix Barbet (CNRS/ENS, Paris), Alejandro Langa (Coruña del Conde), Salvador Domingo Mena (Diputación de Burgos), Marta Negro (Museo de Burgos) e Isabel Rodá (Instituto Catalán de Arqueología Clásica, Tarragona) por facilitarnos información necesaria para nuestra encuesta. A lo largo del artículo se remite con frecuencia a Hispania Epigraphica Online ( $\mathrm{HEpOI}$ ), que se puede consultar en $\langle$ http://eda-bea.es $\rangle$, donde se ofrecen información y fotografías de los epígrafes citados. 
ESTE TRABAJO DESCRIBE 7 inscripciones encontradas en Clunia Sulpicia, Hispania Citerior. Las ruinas de la que fue caput Conventus cluniensis se encuentran sobre el Alto de Castro, una extensa y elevada mesa cuya jurisdicción se la reparten actualmente Coruña del Conde y Peñalba de Castro (una pedanía de Huerta de Rey), en la provincia de Burgos. Aunque conocido desde el s. XVI y primeramente descrito en las últimas décadas del s. XVIII, este extenso yacimiento arqueológico tardó aún otra centuria en interesar a la arqueología moderna: la primera exploración científica de la que hay noticia es la de N. Sentenach en I9I2, es decir, tres años antes de que I. Calvo publicase los resultados de lo que hasta ahora se tenían por las excavaciones iniciales del lugar ${ }^{2}$. A comienzos de los años treinta del pasado siglo, B. Taracena, entonces director del Museo Numantino de Soria y conocedor de las cerámicas locales, se hizo cargo del sitio y entre i932 y I935 excavó una gran estructura edilicia que calificó de "palacio»; desgraciadamente, la empresa se vio truncada de forma dramática e inesperada por la Guerra Civil, lo que también acarreó la carencia de una publicación sistemática3; con todo, Taracena ha sido el responsable del posterior interés por el yacimiento, lo que seguramente explica que en I958, la Diputación Provincial de Burgos encargase a P. de Palol la custodia y el estudio de la antigua ciudad, un empeño que sigue vivo y boyante*.

Clunia Sulpicia ofrece, además, un considerable número de inscripciones; algunas, ya desaparecidas, fueron descritas por quienes como J. Uztarroz, A. Morales y J. A. Loperráez, visitaron el lugar a partir de mediados del siglo XVI; muchas otras están aún a la vista en los edificios de Peñalba y Coruña, procedentes del uso de las ruinas antiguas como cantera de material de construcción; y hay, por último, unas pocas encontradas durante los reconocimientos y excavaciones arqueológicas. Desde hace treinta años se dispone de un excelente catálogo ${ }^{5}$, pero que contiene lecturas erróneas y precisa incorporar los nuevos hallazgos realizados tras su publicación ${ }^{6}$.

2. Vid. Rodríguez Ceballos, Mariano, Gómez-Pantoja, Joaquín L., y Fasolini, Donato: «Arqueología de archivo: La campaña de Narciso Sentenach en Clunia (1913)», Oppidum, 10, 2014, pp. 91-106; CALvo SÁNCHEZ, Ignacio: «Excavaciones en Clunia», Memorias de la Junta Superior de Excavaciones y Antigüedades, 1, 1916, pp. 3-32.

3. Vid. Taracena Aguirre, Blas: «La cerámica de Clunia», Anuario de Prehistoria Madrileña, 2-3, 1931-1932, pp. 85-91; «El palacio romano de Clunia», Archivo Español de Arqueología, 62, 1946, pp. 29-69. Recientemente se ha dado a conocer parcialmente el contenido de los Cuadernos de excavaciones de esa campaña, vid. Hoyo, Javier del, y Rodríguez Ceballos, Mariano: «Epigrafía de Clunia (Burgos) en los cuadernos de excavación de Blas Taracena», Espacio, Tiempo y Forma, 27, 2014, pp. 121-135.

4. Vid. Palol, Pedro de : Clunia 0. Studia Varia Cluniensia, Valladolid, 1991; Langa Aguilar, Alejandro: Colouniocu Clunia = Coruña del Conde, Burgos, 2009; Tuset BertráN, Françesc y De LA IgLesIA, Miguel Ángel (2010) : «Clunia, centro de poder territorial», Actas del Coloquio Internacional Patrimonio Cultural y Territorio en el Valle del Duero: Zamora, 28, 29 y 30 de marzo de 2007, AA.VV. (eds.),Valladolid, 2010, pp.75-85.

5. PAlol, Pedro de y VILELLA, José: «Clunia II. La epigrafía de Clunia», Excavaciones Arqueológicas en España, 105, Madrid, 1987.

6. A guisa de ejemplo y limitándonos a lo publicado en los últimos diez años, vid. GUtiÉrRez BeHEMERID, M. Ángeles et al. (2006) : «El Teatro de Clunia. Nuevas aportaciones», Jornadas sobre Teatros Romanos en Hispania, Cordoba 2002, Márquez, Carlos y Ventura Villanueva, Ángel (eds.) Córdoba, 2006, p. 291; Beltrán Lloris, Francisco y DíAz ARIÑo, Borja : «Altares con téonimos hispano-célticos de la Meseta Norte (Museos de Palencia, Burgos y Valladolid)», Auf den Spuren keltischer Götterverehrung. Aktes des 5. F.E.R.C.AN Workshop. Graz 9-12 Oktober 2003, M. HaInzMANN (ed.), Wien, 2007, pp. 29-56; IRUjo LizAUR, Javier : «Sellos sobre material latericio de la Legio IIII Macedonica en la Colonia Clunia Sulpicia», Oppidum, 4, 2008, pp. 99-112; GIL, Juan : «Fichas epigráficas», Habis, 39, 2008, pp. 369-375; GómEZ-PANTOJA, Joaquín L.: Epigrafia anfiteatrale dell'Occidente romano, VII: Baetica Lusitania Hispania Citerior, Roma, 2009, p. 35; ID.: «Un vaso celtibérico con problema», Cuadernos de Arqueologia de la Universidad de Navarra, 18, 2010, pp. 177-199; González Rodríguez, M. Cruz y Gorrochategui: «Nuevas lecturas 
Casi todas las piezas descritas seguidamente corresponden a la categoría de las inéditas y de las otras dos se completan y mejoran las ediciones disponibles.

\title{
1. GRAFFITI
}

A fines de 2012 y por gentileza de la Dra. I. Rodá, uno de nosotros tuvo la oportunidad de examinar el llamado «Fondo Palol», el archivo personal de interés arqueológico que P. de Palol cedió al Instituto Catalán de Arqueología Clásica de Tarragona. Como es lógico, los papeles relacionados con las excavaciones y la investigación de Clunia ocupan un lugar importante en ese repositorio y, entre ellos, estaba la fotografía de un fragmento de estuco esgrafiado, con una nota manuscrita en el reverso: Clunia, graffiti fond blanc; y la estampilla Photo Alix Barbet / Reproduction interdite. No encontramos, sin embargo, la obligada carta de remisión ni ningún indicio de cómo y cuándo llegó la fotografía a manos del Prof. Palol. Afortunadamente, una búsqueda en Internet nos llevó a la Dra. Alix Barbet, investigadora de la UMR 8546 (CNRS/ENS, Paris) y reputada especialista en pintura mural romana. A nuestra indagación per litteras (5-I2-2012) amablemente respondió informándonos de las circunstancias en que tomó las fotos, a la vez que nos enviaba la imagen de otro grafito de la misma procedencia, del que no nos consta que haya copia en el «Fondo Palol»:

\begin{abstract}
Infatti ho ritrovato la fotografia e il negativo del graffito che l'interessa, ma purtroppo non ho mai avuto la localizzazione precisa. C'è ne un altro e posso numerare tutte due e mandarle (76/15/5 et 76/16/7). Le foto sono state fatte in 1976, e la foto che precede (76/15/6) di frammenti di pittura viene dalla casa 2, ma non è una prova per la provenienza dei graffiti. Ho dovuto convincere il prof. de Palol che i frammenti dovevano essere protetti in cassa e non lasciate per terra come rifuti! ...Dunque non so dove sono andati questi pezzi. Non sono mai ritornata a Clunia; ho cercato da aiutare una studente che voleva specializzarsi in pittura mai dopo un po di tempo, non ho avuto nessuna notizia.
\end{abstract}

Estas dos fotografías despertaron nuestro interés por los graffiti clunienses, de los que se sabe muy poco pero que, a la vista de las noticias de la Dra. Barbet, de lo

\footnotetext{
de tres inscripciones de Clunia (Hispania Citerior) con repercusión sobre la expresión de algunas agrupaciones familiares celtibéricas.», Veleia, 28, 2011, pp. 269-280; Balbín Chamorro, Paloma, Sevillano López, David y Rodríguez Ceballos, Mariano: «Nuevo fragmento de ara funeraria de Peñalba de Castro (Burgos) y un posible taller epigráfico en Clunia», Ficheiro epigráfico, 108, 2013, 478; Hoyo, Javier del, y Rodríguez Ceballos, Mariano: op. cit. pp. 121-135; Sevillano López, David y Rodríguez Ceballos, Mariano: «Nueva inscripción funeraria en Peñalba de Castro / Burgos», Ficheiro epigráfico, 123, 2014, 525; GutIÉRrez BeHEMERID, M. Ángeles: «Altar funerario con pvlvini de la ciudad de Clunia», Veleia, 32, 2015, pp. 189-198; Hoyo, Javier del, y Rodríguez Ceballos, Mariano: «A tiro de piedra. Nuevos epígrafes de Clunia en Coruña del Conde (Burgos)», Habis, 46, 2015, pp. 107-127; EID.: «Vestigios epigráficos procedentes de «Clunia» en Coruña del Conde (Burgos)», Cuadernos de arqueología de la Universidad de Navarra, 23, 2015, pp. 255-278; ABASCAL PALAzón José Manuel.: «La ermita del Santo Cristo de San Sebastián (Coruña del Conde, Burgos) y sus monumentos de época romana», Archivo Español de Arqueología 88, 2015, pp. 223-246.
} 
publicado $^{7}$ y de nuestras indagaciones, parecen haberse hallado en relativa abundancia, tanto durante las excavaciones de Taracena como en las campañas posteriores de Palol. Desgraciadamente, de los cuatro letreros que damos a conocer, sólo hemos podido examinar el depositado en el Museo de Burgos; los restantes, los describimos a partir de sus imágenes. Todos pueden considerarse inéditos, al menos en lo referente a sus lecturas.

\subsection{FRAGMENTO DE ESTUCO DE LA TABERNA 8}

Es el único del que hay noticia impresa y se encontró al pie de la pared meridional del llamado «ambiente número 8» que los excavadores del foro cluniense identificaron como «una sala de culto, aunque seguimos llamándola taberna». La relación de lo hallado en ese lugar contiene un dibujo del grafito, acompañado de sendas interpretaciones del mismo ${ }^{8}$. Consta también que el esgrafiado lo hicieron manos distintas sobre el estuco rojo del muro sur de la estancia, siendo a) el texto más largo y legible, con letras capitales rústicas de tamaño uniforme y más pequeñas que los otros dos; en cambio, b) se superpone sobre la línea 6 de a), enmascarando o aprovechando algunos de sus rasgos; y c) está separado de los dos anteriores, se arañó con las letras más grandes y más difíciles de identificar porque contienen lo que parecen nexos imaginativos. Nada se dice, en cambio, sobre las dimensiones de los letreros y su letras y ni del paradero de este grafito y de los restantes encontrados con él; tampoco se ofreció lectura del mismo, aunque I. Velázquez añadió una a la noticia de su hallazgo en HEp ro, 2000, 96.

Como suele ser habitual, resulta difícil entender el sentido de estos letreros y más si están incompletos; pero la posible secuencia puerum [- - -?] praetexta[um] recuerda los sueños premonitorios de Q. Catulo sobre Augusto (Suet., divus Aug. 94, 8-9) y, decididamente, la referencia a camilli no resulta impropia en una estancia que sus excavadores juzgaron de funcionalidad religiosa por su planta singular, por el tenor del material arqueológico encontrado y por su cercanía al templo situado en el extremo meridional de foro. Éste, desde su descubrimiento a comienzos del siglo XX, ha venido asignándose al culto Júpiter, dado el papel que el sacerdos Iovis cluniense jugó en el pronunciamiento imperial de Galba (Suet. Galba, 9.2). Estuviera o no consagrado al Padre de los dioses, de lo que no cabe duda es que su devoción debió gozar de especial relevancia en la capital conventual`9.

7. Entre los resultados de las excavaciones del foro local (PALOL, Pedro de y GUitart Durán, Josep: Los grandes conjuntos públicos: el foro colonial de Clunia. Clunia VIIl.1, Burgos, 2000) se señala el hallazgo en una de sus dependencias (vid. infra) de «un conjunto importante de restos del estucado del muro, pintado de rojo, con figuraciones de edificios, quizás un templo muy borrado, y algunos grafitos muy fragmentados arañados sobre el rojo, que tenemos en estudio».

8. Palol, Pedro de y Guitart Durán, Josep, op. cit. pp. 71 y 75 y figs. 31-32.

9. El más completo análisis de los vestigios del templo del foro en GutiérRez BehEmerid, M. Ángeles y Subías Pascual, Eva: «El llamado Templo de Júpiter de Clunia», Archivo Español de Arqueología, 73, 2000, pp. 147200, cuyas autoras se muestran escépticas sobre la posibilidad de determinar su advocación a partir de los restos arquitectónicos. Sobre el episodio cluniense de Galba, vid. HALEY, Evan W. : «Clunia, Galba and the events of 6869», Zeitschrift f. Papyrologie u. Epigraphik, 91, 1992, pp. 159-164. 
Nuestra transcripción de los tres dibujos es:

a)

rebu[s? - - ]

dixit $[---]$

P puerum $[--$ - $]$

praetextạ[- - - ]

$5^{-} \quad \operatorname{sexen}+[---]$

NṬN T[- - ]

b) $\mathrm{S} \mathrm{V}$

c) NT FE RD

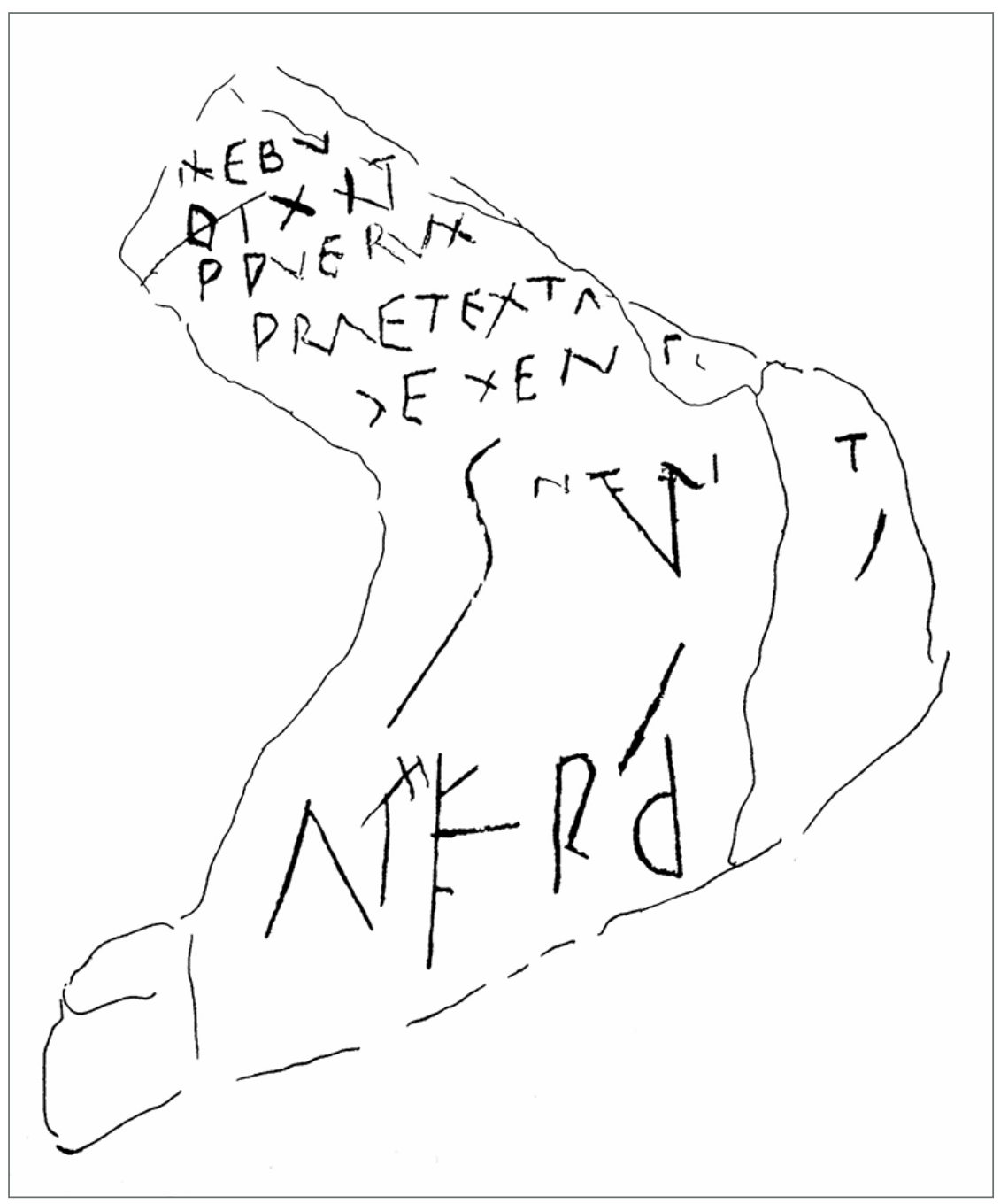

FIGURA 1: CALCO DEL GRAFITO 1.1., SEGÚN PALOL Y GUITART.

a) I-3: om. Palol y Guitart, fig. 32b - $3 p$ dueru+ Velázquez ad HEp - 5 sexens

Palol y Guitart, fig. $32 \mathrm{a}-6 \mathrm{om}$. Palol y Guitart, fig. $32 \mathrm{a}-\mathrm{c}$ ) MERD Palol y Guitart, fig. $32 \mathrm{a}$ - NERD+ sexens Palol y Guitart, fig. 32b. 


\subsection{FRAGMENTO DE ESTUCO BARBET 1}

Como ya se ha dicho, el letrero se conoce gracias a la fotografía existente en el «Fondo Palol» del Instituto Catalán de Arqueología Clásica y corresponde al negativo 76/15/7 del archivo fotográfico de la Dra. Barbet, quien amablemente nos proporcionó la copia que reproducimos. Se trata de un fragmento de estuco blanco, de forma aproximadamente trapezoidal y cuyas dimensiones son $(\mathrm{I} 3,3) \mathrm{x}$ (II) $\mathrm{cm}$. Contiene cuatro renglones mutilados por la derecha y de dos manos distintas, a juzgar por el tamaño de las letras: los rasgos de la primera línea miden 3,7 $\mathrm{cm}$, mientras que la altura de los dos siguientes es I,2-I,3 cm; las letras de la cuarta están rotas por la mitad y resultan difícilmente identificables. Visto y fotografiado por la Dra. Barbet en Clunia, en I976, posiblemente en la llamada Casa 2. Desconocemos su paradero actual.

a)

$$
\begin{aligned}
& ++ \\
& \mathrm{X}[---]
\end{aligned}
$$

b) $\quad \Theta E[---]$

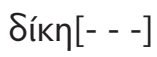

$++$
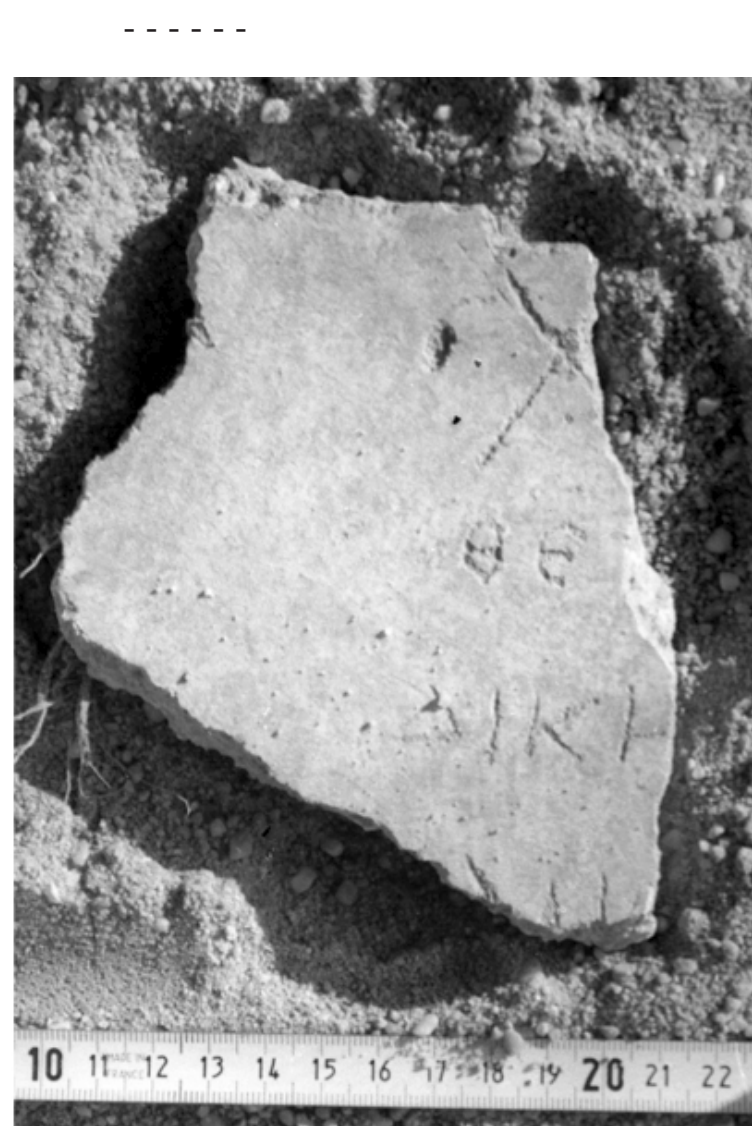

FIGURA 2: GRAFITO 1.2. (C) Alix Barbet 


\subsection{FRAGMENTO DE ESTUCO BARBET 2}

Este grafito se describe también a partir de otra fotografía amablemente cedida por la Dra. Barbet y procede del negativo $76 / 15 / 5$ de su archivo fotográfico. Se trata de tres minúsculos fragmentos contiguos de estuco blanco y que juntos miden $($ I 3,5$)$ x (I7) cm. Se ven dos renglones de texto, de manos distintas La primera línea contiene dos secuencias, la de la izquierda medio borrada, ya que sólo quedan los rasgos inferiores de algunas letras cursivas; el resto sin embargo, conserva las letras íntegras, que son de factura irregular, pues miden entre 3,8 y I cm. En el segundo renglón sólo quedan los rasgos de dos numerales, uno de ellos muy mutilado ( $\mathrm{I}, 4 \mathrm{~cm})$. Visto y fotografiado en Clunia en las mismas circunstancias que el fragmento anterior.
a)$$
[---][[+++]] \operatorname{eocr}+\mathrm{a}[---]
$$$$
\text { b) } \quad[---] X X[---]
$$

En a), la letra dudosa puede ser una $\mathrm{R}$ o una $\mathrm{H}$; la parte legible posiblemente corresponde a la terminación de un nombre griego como Neocrates, Theocrates o, si se tratase de una $\mathrm{H}$, Teocharis.

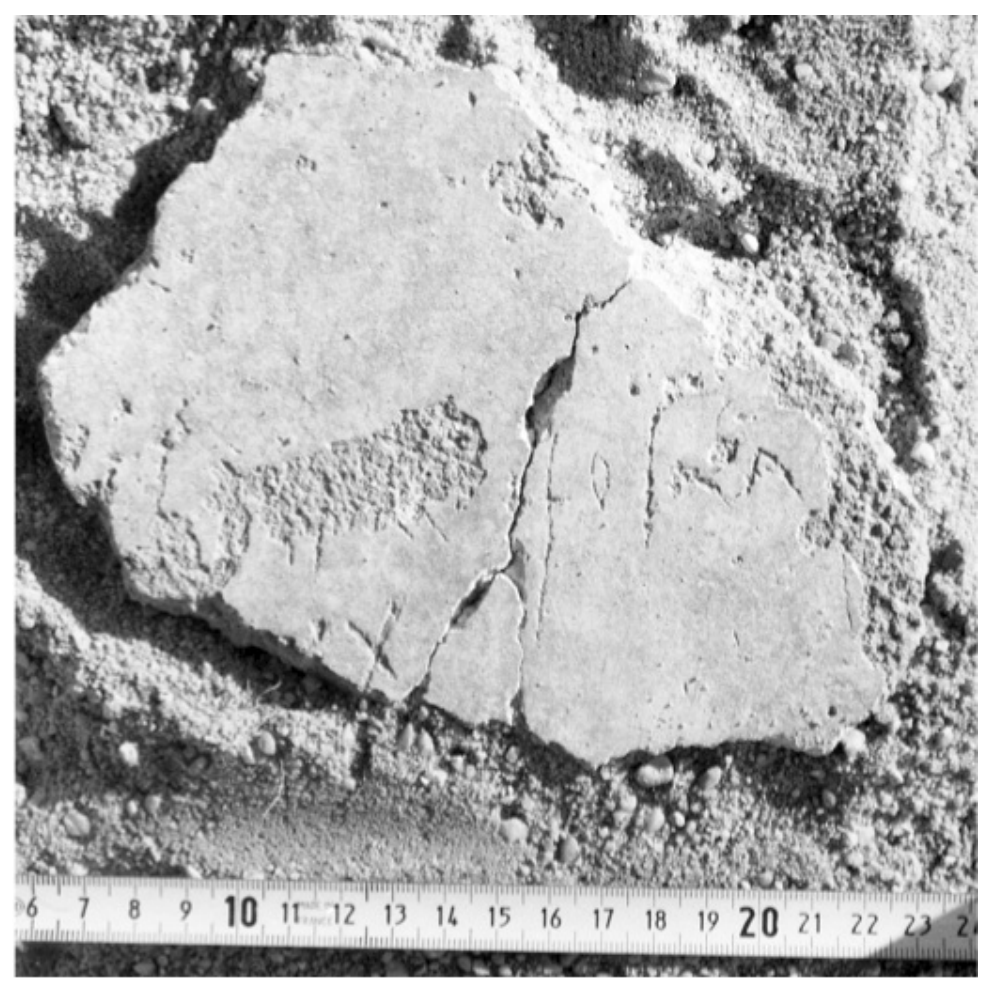

FIGURA 3: GRAFITO 1.3. (C) Alix Barbet. 


\subsection{FRAGMENTO DE ESTUCO EN EL MUSEO DE BURGOS}

Este pequeño fragmento de forma irregular de estuco pintado como crusta marmorea verde con vetas negras mide $(7) \times(6) \times(\mathrm{I}, 8) \mathrm{cm}$; letras, $\mathrm{I}, 3 \mathrm{~cm}$. Forma parte parte de la exposición permanente del Museo de Burgos y es la única pieza de esta clase conservada en el conjunto de los materiales procedentes de las excavaciones de B. Taracena en Clunia. Fuera de su origen y la implícita fecha de su descubrimiento (I932-35), se desconoce cualquier otra circunstancia de su hallazgo.

$$
\begin{aligned}
& - \text { - - - -? } \\
& - \text { - -]AN+[- - } \\
& \text { - - - ]+Kaí Y[- - - } \\
& \text { - - -]XOLA[- - }
\end{aligned}
$$

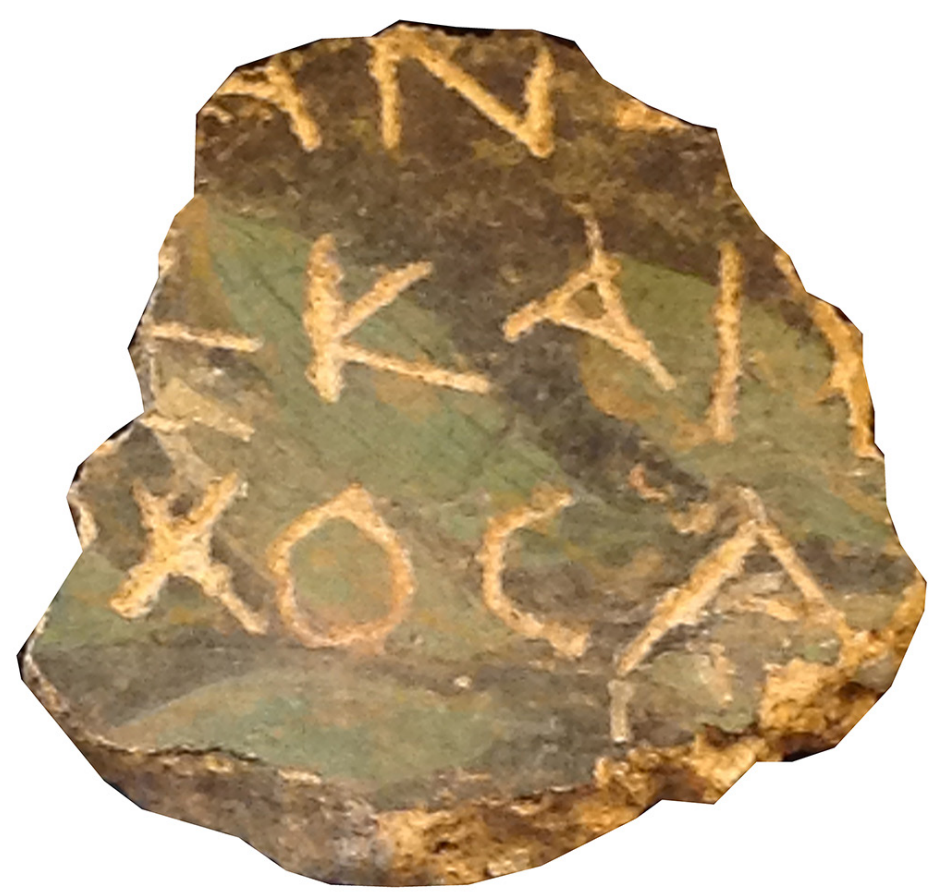

FIgURA 4: GRAFITO 1.4. (C) Museo de Burgos y Mariano Rodríguez.

\section{SPOLIA VARIA}

\subsection{FRAGMENTO EPIGRÁFICO CON MENCIÓN DE TRIBU}

Sin que haya constancia de su lugar de hallazgo ni de las circunstancias del mismo, también forma parte de los materiales excavados por Taracena el fragmento epigráfico que se describe a continuación y cuya procedencia es segura por haber 
estado depositado en el Museo Numantino antes de su traslado al Museo de Burgos a comienzos de 1980. El inventario de esa mudanza permitió a Palol y Vilella dar noticia del mismo: [- - ] / [- - ]QU[- - -] / [- - ]BUR[- - - / [- - ]XX[- - - / [- - - ] $]^{\text {10 }}$.

Aún con la parvedad de la información disponible, sin haberlo visto por desconocer su paradero, hicieron notar que lo suponían parte de un epitafio. La revisión sisemática del Lapidario del Museo de Burgos que hemos realizado en pasados años nos ha permitido localizar la pieza en sus almacenes. Se trata de una porción irregular de un bloque de piedra caliza, roto por todos sus lados y cuyas medidas actuales son (29) x (I9) x (I5) cm. Del epígrafe apenas quedan ocho o nueve letras, que son capitales cuadradas de buena factura y que miden $6 \mathrm{~cm}$. A partir de lo conservado, proponemos la siguiente restitución:

FIGURA 5: FRAGMENTO 2.1. (C) Museo de Burgos y Mariano Rodríguez.
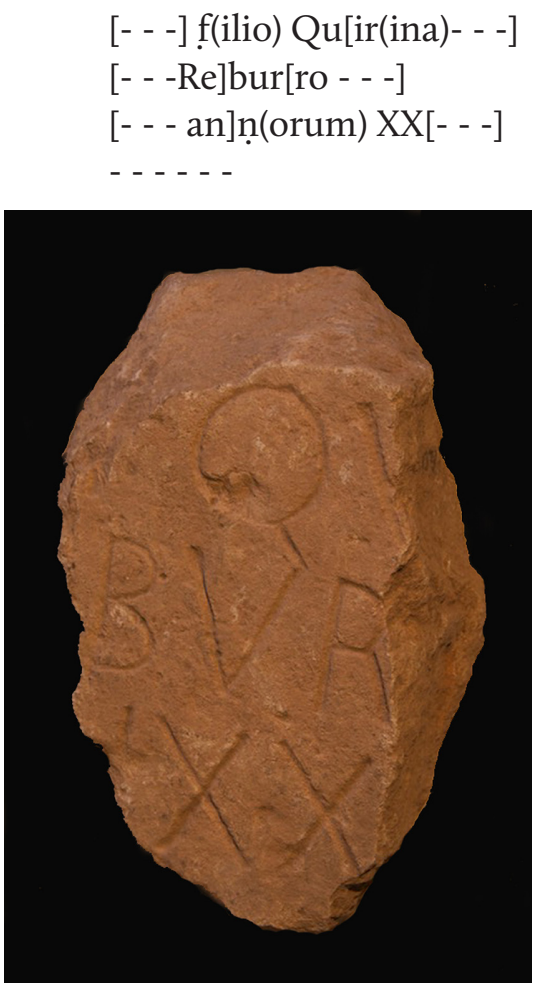

Al comienzo de la primera línea se aprecian los ápices de dos brazos que deben corresponder a la sigla de la filiación, lo que permite identificar los rasgos siguientes como el inicio de la mención tribal y las tres letras de la línea segunda como pertenecientes a uno de los cognomina más corrientes en la zona ${ }^{\mathrm{Ir}}$; por último, los trazos de la tercera línea refieren obviamente a la edad del difunto.

La tribu de los ciudadanos de la colonia Sulpicia fue la Galeria, pero allí están atestiguados también otros tribules, entre ellos un notable grupo de individuos adscritos a la Quirina. No debe sorprender esta circunstancia considerando que se trata de la capital de un conventus en su mayor parte municipalizado a partir de la época flavia ${ }^{\text {I2 }}$.

10. Palol, Pedro de y Vilella, José: op. cit. p. 112, a partir del inventario de los materiales transferido por el Museo de Soria al de Burgos en virtud de la Resolución del Ministerio de Cultura de 28 de febrero de 1980, Archivo del Museo de Burgos.

11. Ciertamente Reburrus no es la única reconstrucción posible pero sí la más probable, dado que está atestiguado en la propia Clunia y fue muy popular en la Hispania céltica.

12. Vid. KUBITSCHEK, Joseph W.: Imperium romanum tributim discriptum, Praga, 1889, p. 192; WIEGELS, Rainer: Die Tribusinschriften des römisches Hispanien. Ein Katalog, Berlin, 1985, pp. 106-108; FAsolıNI, Donato: Le tribù romane della Hispania Tarraconensis. L'ascrizione tribale dei cittadini romani nelle testimonianze epigrafiche, Milano, 2012, pp. 249-265. Los testimonios de la Quirina en Clunia son CIL II $2798=$ HEpOl 8624 (Peñalba de Castro); HEpOl 25691 (Vivar del Cid); CIL 2802 = AE 1981, $554=\mathrm{HEpOl} 8628$ (Alcubilla de Avellaneda); AE 1990, 563 = HEp 3, 1993, 111 = HEp 4, 1994, $197=\mathrm{HEpOI} 15304$ (Contreras de Burgos). Sobre la municipalización del Conventus cluniensis, vid. ANDREU 


\subsection{PORCIÓN DE UN PEDESTAL O ARQUITRABE}

Paralelepípedo de caliza local cuyas dimensiones son (44) x (99,5) x $64 \mathrm{~cm}$. Salvo por alguna herida y la degradación superficial, el bloque está bien preservado, pero es claro que lo que se conserva es solo una parte de un elemento arquitectónico mayor, como demuestra la divergencia entre la arista de la lastra y la línea base del epígrafe, la posición asimétrica de éste en la cara inscrita y el que el texto, palmariamente incompleto, ocupe toda la anchura del fragmento pétreo: de ahí que supongamos que se trata de parte de un pedestal o dintel.

De la inscripción sólo resta una sola línea con siete letras de buena factura y 7,5 $\mathrm{cm}$ de altura, que ocupan toda la anchura de la pieza, lo que es un argumento más en apoyo de que la inscripción está mutilada. Sirve de jamba de la puerta de una tenada o henil existente a las afueras de Peñalba de Castro, al pie de la ladera norte del Alto de Castro. Vista, descrita y fotografiada en varias ocasiones entre 2007 y 20I4. Lo que se lee en la piedra es:

- - -in] h(onorem) A(uli) Stric̣[onis- - -

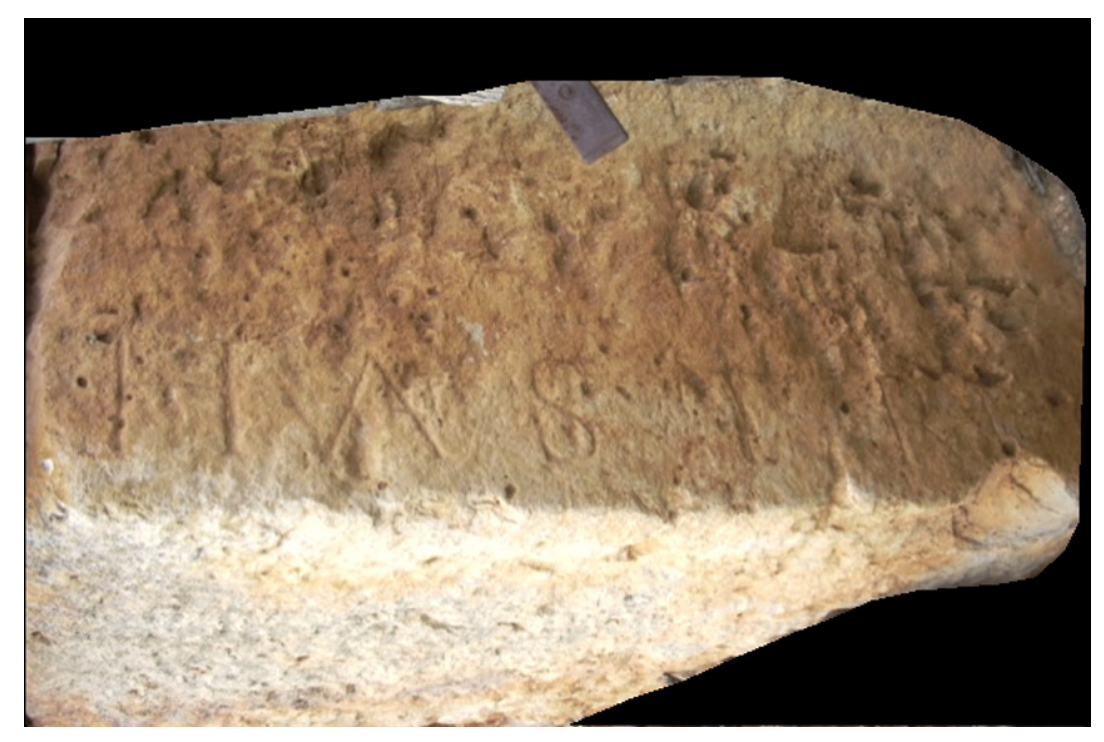

FIGURA 6: FRAGMENTO 2.2. (C) Joaquín L. Gómez-Pantoja.

Antes de la A hay un rasgo menos nítido que sugiere un nexo VA; sin embargo, la incisión es menos profunda que en las otras letras, no guarda la medida de línea y tampoco hace más inteligible el texto, por lo que lo suponemos un rasguño accidental o un añadido moderno. Mayor transcendencia tiene la ambigua lectura de la última letra, porque al no apreciarse con claridad su remate bajo, puede ser una C o una G; en esas circunstancias, el infrecuente Stric[tus] es una posible

PINTADO, Javier: «Apuntes sobre la Quirina tribus y la municipalización flavia de Hispania», Revista Portuguesa de Arquéologia, 7(1), 2004, pp. 343-364. 
restitución, pero Strigo está ciertamente mejor documentado, existiendo incluso la variante Strico $^{13}$.

\subsection{FRAGMENTO DE EPITAFIO}

Bloque de caliza local de grano fino, que mide (26) x (32) x (29) cm; se trata de un fragmento de forma irregular, muy mutilado, procedente de un monumento ortoédrico del que se conserva parcialmente la cara inscrita y el costado derecho, habiéndose perdido un número indeterminado de líneas por arriba y abajo; de las cuatro aún visibles, la primera y la última son las peor conservadas; letras capitales de módulo alargado $(6 \mathrm{~cm})$ y trazo curvilíneo. Como lo que resta del letrero es apenas inteligible, debe suponerse que se trata posiblemente del fragmento del costado derecho de un cipo de grosor apreciable, de los que hay varios ejemplos entre las inscripciones locales. Encontrado por uno de nosotros en Peñalba de Castro, en un vertedero de escombros a los pies del Alto de Castro y seguramente desechado tras haber servido como material de construcción en alguna casa del pueblo. Tras su hallazgo, se entregó al Museo de Burgos, donde se conserva sin inventariar. Lo que se lee en la piedra es:

$$
\begin{aligned}
& \text { - - -l]eg · v[II] } \\
& \text { - - -]natus } \\
& \text { - - -ve]țeranus • vị- } \\
& \text { - - -par]ẹntiḅ[us- c.2 -] } \\
& \text { - - - - - - }
\end{aligned}
$$

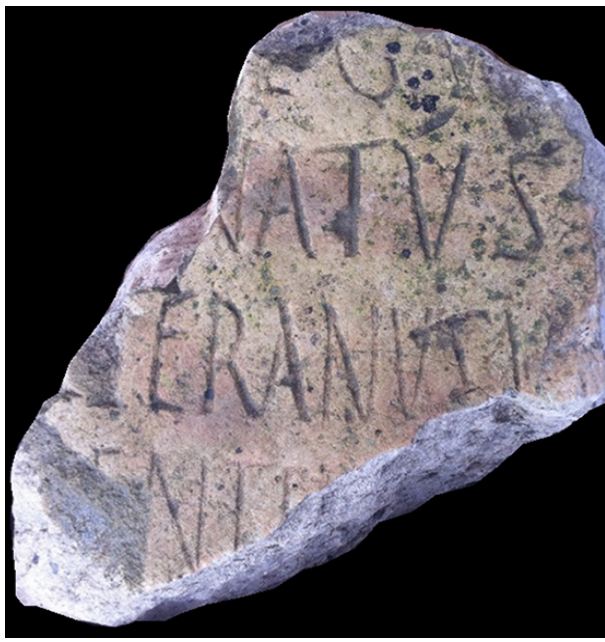

FIGURA 7: FRAGMENTO 2.3. VISTA GENERAL (C) Mariano Rodríguez.

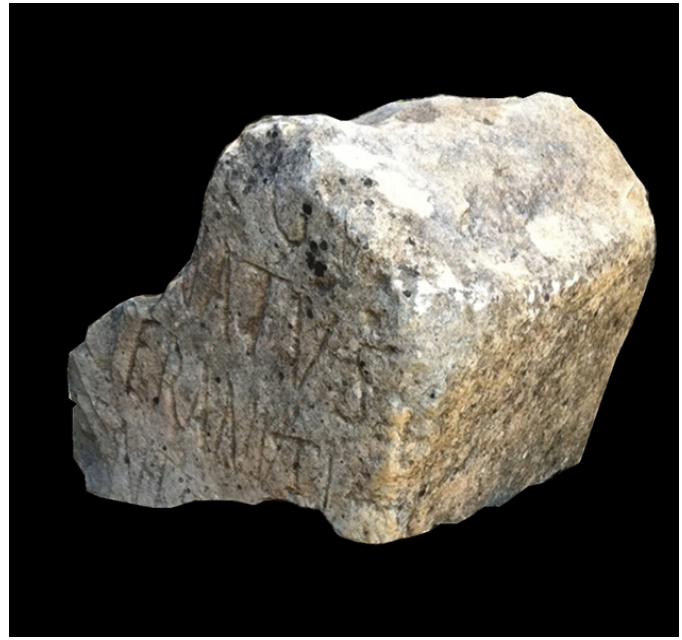

FIGURA 8: FRAGMENTO 2.3. DETALLE (C) Mariano Rodríguez.

13. Strictus; AE 1929, 164 = Giuliano, A. (ed.): Museo Nazionale Romano. Catalogo Generale. I, 7-1, Le sculture, Roma, 1984, p. 138, de Trebula Metuesca. Strigo: además del famoso M. Fufius M.f. Strigo (vid. PIR² F 513), hay sendos individuos mencionados en CIL VI 1622 y 33027, de Roma, donde está también atestiguada la variante Strico, CIL VI 21358. 
Dada la fragmentación del monumento y la imposibilidad de conocer las dimensiones de la parte perdida, la restitución del texto es azarosa. En la primera línea, la secuencia conservada hace obvia la referencia a la legión hispana por excelencia, habiendo espacio suficiente para completar la parte final de numeral; la legio VII Gemina fue constituida en Clunia por Galba y están atestiguados un número significativo de milites de esa procedencia sirviendo en legiones acuarteladas en diversas provincias del Imperio y, entre ellos, tres enrolados en la propia leg. VII ${ }^{14}$.

En los renglones siguientes, [ve]teranus y [par]entib[us] parecen lecturas seguras, mientras que el vi- al final de la tercera línea puede ser el comienzo de vixit o vivus. La mayor duda reside en el segundo renglón, donde la secuencia legible puede entenderse como parte de un nombre personal (Donatus, Fortunatus, menos probable Cincinatus) o parte de la locución [donis do]natus. La primera alternativa parece la más común pero exige que el epitafio mencione, además de a los padres, al menos a dos o más individuos, siendo el último el autor y dedicante del monumento. La segunda opción, en cambio, resulta en la ejecutoria militar de un único individuo, que hizo la tumba para sí y sus padres. Nos inclinamos por esta segunda hipótesis, por lo que en líneas generales, lo que queda del epígrafe podría restituirse [- - - - - / - - -miles l]eg(ionis) · V[II / - - - donis do]natus / - - ve]teranus · vi/[vus sibi et par] entib[us fecit - - - - - ].

Aunque como se ha hecho notar a lo largo de este trabajo, ninguna de las siete piezas editadas apenas tienen de por sí otro valor que acrecentar el futuro catálogo epigráfico de Clunia Sulpicia, desde una perspectiva más amplia, muestra también que el lugar sigue siendo del mayor interés epigráfico.

14. Reclutas de esta procedencia constan en la legiones I Italica (AE 1999, 1333); III Augusta (CIL VIII 2807); la VIIII Hispana (CIL VII 184) y la XIIII Gemina Martia Victrix (CIL III 1158; CIL XIII 6911), más el [Fla?]vius Reburrus conmemorado en el Tropaeum Traiani y cuya unidad se desconoce (CIL III 14124). Sobre los tres Septimani, vid. PALAO VICENTE, Juan José : Legio VII Gemina (Pia) Felix. Estudio de una legión romana, Salamanca, 2006, passim. 


\section{8

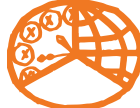 \\ ESPACIO, \\ TIEMPO \\ Y FORMA}

Prf. Dr. José Mª Blázquez Martínez, In Memoriam

\section{Artículos · Articles}

19 CÉsAr Fornis

Bajo el signo de Licurgo: el reformismo atávico de Agis IV y Cleómenes III / Under the sign of Lycurgus: the atavistic reformism of Agis IV and Cleomenes III

\section{9}

Jorge García Sánchez \& Antonio López García

Las bibliotecas del Oriente romano como Heroa. Evergetismo cultural y propaganda familiar / Eastern Roman Libraries as Heroa. Cultural evergetism and family propaganda

65

YANN LE BOHEC

Conueteranus, -i, et les solidarités militaires / Conueteranus, $-i$, and military interdependences

\section{5}

Helena Gozalbes García

La corona cívica en la moneda provincial de la Hispania romana / The civic wreath in the provincial coinage of the Roman Hispania

\section{7}

NARCISO SANTOS YANGUAS

La inscripción de Pentio Flavio hallada en Corao (Cangas de Onís) y los vadinienses del oriente de Asturias / Pentio Flavio's inscription found in Corao (Cangas de Onís) and the vadiniense population from orient Asturias
10 joaquín L. Gómez-Pantoja \& Mariano Rodríguez Ceballos \& DONATO FASOLINI

Minima Epigraphica Cluniensia / Minima Epigraphica Cluniensia

\section{Consol García Ribot I Serra}

Nuevos Testimonios del culto a Cibeles-Attis en la província de Barcelona (España) / New testimonies of the cult to Cibeles-Attis in the provincial of Barcelona (Spain)

\section{José Ma Blázquez Martínez (†)}

La Traditio Legis de Cristo a Pedro y Pablo en un plato de vidrio de Cástulo, Linares (Jaén) / Traditio Legis of Christ to Peter and Paul in a glass bowl from Cástulo, Linares (Jaén)

\section{Enrique Gozalbes Cravioto \\ Visiones del África Romana / Views of Roman Africa}

\section{Reseñas · Book Review}

Bravo Jiménez, Salvador: Control ideológico y territorial en el Estrecho de Gibraltar en la Antigüedad (Siglos X-I a.C.) (Marta Bailon García).

183 Azcarraga, Cámara, Sandra: El ocaso de un pueblo. La Carpetania centro-septentrional entre la segunda Edad del Hierro y la época romanana (Siglos III A.C.-I D.C.) El valle bajo del Henares (PILAR FERNÁNDEZ URIEL). 